\title{
Research on the Application Mode of Green Environment Design under the Background of Artificial Intelligence
}

\author{
Hui Liu $\mathbb{D}^{\mathbb{D}},{ }^{1}$ Guanghui Song $\mathbb{D}^{1},{ }^{1}$ and Linlin Yan $\mathbb{D}^{2}$ \\ ${ }^{1}$ School of Architectural \& Artistic Design, Henan Polytechnic University, Jiaozuo, Henan 454000, China \\ ${ }^{2}$ Jiaozuo Branch of China United Network Communications Group Co., Ltd., Jiaozuo, Henan 454002, China \\ Correspondence should be addressed to Guanghui Song; songguanghui@hpu.edu.cn
}

Received 15 April 2021; Revised 5 May 2021; Accepted 23 May 2021; Published 1 June 2021

Academic Editor: Zhihan Lv

Copyright $\odot 2021$ Hui Liu et al. This is an open access article distributed under the Creative Commons Attribution License, which permits unrestricted use, distribution, and reproduction in any medium, provided the original work is properly cited.

In the field of green environmental design, the design system of environmental art is a complex and multidimensional crossdomain fusion system, which can be regarded as a large task system. The development model that combines artificial intelligence (AI) technology and green environment design can not only integrate and analyze problems quickly and efficiently but also provide designers with new design ideas, gradually extending traditional environment design concepts, producing more diverse artistic creation methods, and shifting from a fixed application model to a more diversified artistic development. Therefore, on the basis of summarizing and analyzing previous research works, this paper expounded the research status and significance of green environment design, elaborated the development background, current status, and future challenges of AI technology, introduced the network topology and platform frame structures of green environment design under the AI background, constructed a technical framework of green environment design under the AI background, analyzed the methods and principles of program design and function development, proposed the application model of green environment design under the AI background, conducted design function model optimization and intelligent design process analysis, and finally discussed the evaluation indicators and hierarchical analysis of the green environment design under the AI background. The results show that the application mode of combining AI technology and green environment design can not only help designers break through traditional time and space barriers and use multidimensional thinking but also help designers have a new understanding of artistic design concept and give full play to the advantages of artificial intelligence in a green environment. The study results of this paper provide a reference for further research on the application mode of green environment design under the AI background.

\section{Introduction}

The development model that combines artificial intelligence (AI) technology and green environment design is determined by the needs of human life under the background of time development. Modern technology can also be used in the green environment design work, which can help designers break through the traditional time and space barriers, use multidimensional thinking to solve problems, and establish a unique design concept in the design industry [1]. The development of science and technology is an inevitable product of the development of the AI era, and the continuous development of green environmental design is determined by the high-quality needs of human beings, and the high-tech, high-ecological, and sustainable design concepts are determined by the development of the times [2]. The environmental design concept of low pollution, low cost, high accuracy, strong applicability, ecological sustainability, and five-in-one environment can provide people with a more comfortable and ecological living environment and allow people to have a better living experience [3]. In the design process, the designer must not only have a certain design concept, plan, and model, but also formulate a series of design cost planning, which is an essential and important link in the design plan. Therefore, it is of great significance to study the application mode of green environment design under the AI background [4].

The green environmental design involves project writing, site inspection, data collection, information arrangement, and scheme selection, with an essentially entire 
process of constant search for answers. In this process, due to the multiple constraints of cost, time, manpower, and material resources, in fact, the problems that people can discover and solve are limited, but AI technology does not have these problems at all [5]. Compared with the designer's limited energy and time, the advantages of the instantaneous reading and writing capabilities of massive computing and massive data of AI are self-evident. With the development of statistics, big data technology has become an effective support for AI technology [6]. The intelligent technologyaided design composed of the two can not only integrate and analyze problems quickly and efficiently, but also integrate data and make decision-making suggestions for designers. Judging from the previous design work promotion mode, the main factors that affect the green environmental design are community location, crowd composition, behavior mode, and sustainable development [7]. Crowd composition and behavior patterns determine the landscape composition, color matching, route planning, and distribution of related facilities in the area. Derivative issues are such as landscape promotion of social interaction and landscape interaction. The sustainable development of the green environment determines the flexible planning of the community space and meets the various needs of community development within a certain period of time [8].

Based on the summary and analysis of previous research works, this paper expounds the research status and significance of green environment design, elaborates the development background, current status, and future challenges of AI technology, introduces the network topology and platform frame structures of green environment design under the AI background, constructs a technical framework of green environment design under the AI background, analyzes the methods and principles of program design and function development, proposes the application model of green environment design under the AI background, conducts design function model optimization and intelligent design process analysis, and finally discusses the evaluation indicators and hierarchical analysis of the green environment design under the AI background. The study results of this paper provide a reference for further researches on the application mode of green environment design under the AI background. The detailed chapters are arranged as follows: Section 2 introduces the network topology and platform frame structures of green environment design under the AI background; Section 3 constructs a technical framework of green environment design under the AI background; Section 4 proposes the application model of green environment design under the AI background; Section 5 discusses the evaluation indicators and hierarchical analysis of the green environment design under the AI background; Section 6 is conclusion.

\section{AI-Based Systematic Structure of Green Environment Design}

2.1. Network Topology Structure. The green environment design system under the AI background should be an innovation of traditional design methods, involving many aspects of the design work of product manufacturers [9]. The network needs to extract useful information from a large amount of stored data, discover the needs and preferences of users from a large amount of user-run data, and then adapt to the needs of users and provide them with flexible and effective services.

For an element whose node $a$ is taken as the starting point and other elements whose distance $b$ from the element has $a$ node of $c$, the probability that two nodes between the two elements occur simultaneously along the direction $Q(a$, $b, c)$ is as follows:

$$
Q(a, b, c)=\frac{\left|W_{a}-E_{b}\right| \cdot\left|W_{c}-E_{b}\right|}{f(a, b)-f(c, b)}=\sqrt{\sum_{a, b, c=1}^{n} \frac{\left[W_{a}-E_{b}\right]^{2}}{\left[W_{c}-E_{b}\right]^{2}}}
$$

where $W_{a}$ and $W_{c}$ are the coordinates of the elements corresponding to nodes $a$ and $c$, respectively; $E_{b}$ is the conversion rule corresponding to distance $b ; f(a, b)$ is the similarity of the matrix of $a$ and $b$ nodes; $f(c, b)$ is the similarity between $c$ and $b$-node matrix; $n$ is the position number of the area.

If the element $R_{i j}$ in the node index layer dominates the elements in the subindex layer $T_{i j}$, decision makers or experts can use the method of pair-wise comparison to reply and then assign the corresponding evaluation scale $\left|R_{x}-T_{x}\right|$ as follows:

$$
\left|R_{x}-T_{x}\right|=\left[\begin{array}{cccc}
\frac{r_{11}}{t_{11}} & \frac{r_{12}}{t_{12}} & \cdots & \frac{r_{1 m}}{t_{1 m}} \\
\frac{r_{21}}{t_{21}} & \frac{r_{22}}{t_{22}} & \cdots & \frac{r_{2 m}}{t_{2 m}} \\
\vdots & \vdots & \cdots & \vdots \\
\frac{r_{m 1}}{t_{m 1}} & \frac{r_{m 2}}{t_{m 2}} & \cdots & \frac{r_{m m}}{t_{m m}}
\end{array}\right],
$$

where $r_{i j}$ is the relative importance of the element $R_{i j}$ compared to $T_{i j}$; $t_{i j}$ is the weight of the subindex $T_{i j}$ to the index $R_{i j} ; \mathrm{m}$ is the number of elements in the index layer $R$.

Suppose that $D_{n}$ is the green environment project to be designed; $\left\{d_{1}, d_{2}, \ldots, d_{n}\right\}$ are the $n$ indicators of the design system; $e_{i}$ is the value of each indicator; $g_{i}$ is the competitiveness of the design project; the green environment design is successful rate evaluation indicators system that can be organized as

$$
D_{n}=\frac{\sum_{x=1}^{n}\left(d_{x}-e_{x}-g_{x}\right)}{\left|R_{x}-T_{x}\right|} .
$$

Green environmental design should combine the product's environmental impact and resource and energy consumption in the use and maintenance phase, feed back related product use information, so as to continuously improve and update product design, and finally form an organic advanced design system. The planning and 
reasoning in AI studies how the machine automatically generates a series of actions and commands to complete the given task under a given goal and task and how to observe the user when the information is incomplete behaviors, understanding their intentions.

2.2. Platform Frame Structure. Through the analysis of the green index in the subindex layer, it is found that there is actually an inherent connection between the evaluation indicators of the environmental design green index, and the five main index evaluations can be further screened out to measure the quality of the green index of the project. Based on the definition of the similarity of the corresponding elements of different matrices, the definition of the similarity $U(x)$ between the two matrices can be further given:

$$
U(x)=f(x)[I(x)-O(x)],
$$

where $f(x)$ is the type of evaluation indicators of green environment design project $x ; I(x)$ is the competitiveness level of green environment design project $x ; O(x)$ is the intelligence index of green environment design project $x$. If $h$ represents the green evaluation weight vector, the green evaluation coefficient $P_{h}$ of the success rate of environmental design projects can be calculated as follows:

$$
P_{h}=\int_{h=1}^{n} U(h) \frac{[k \cdot u(h)-l \cdot v(h)]}{w(h) \cdot z(h)},
$$

where $k$ is the green evaluation weight; $l$ is any secondary index; $u(h)$ is the set of green evaluation weight vectors; $v(h)$ is the risk assessment function of green environmental design; $w(h)$ is the risk of green environmental design evaluation matrix; $z(h)$ is the average random consistent index.

Such intelligent lighting, in addition to helping people avoid light pollution, can also help people get along with nature and play a positive role in alleviating people's psychological pressure. In addition, most of the green environment design is based on green plants and public facilities, while the artificially intelligent green environment can realize resource sharing, etc. and complement each other with local humanities and realize the organic combination of multiple benefits. Green environment design in the era of AI can no longer simply consider human-computer interaction. It should take consciousness, emotion, ethics, aesthetics, and even the society and culture of humans as the starting point and deeply consider the relationship between humans and machines.

The progress $A_{i}$ of $\mathrm{AI}$ information processing can be obtained by using the ratio of the number of times the information is automatically processed in each circulation link of the intelligent node to the total number of information processing times and then multiplying it by the sum of the importance of each intelligent node:

$$
A_{i}=\frac{\beta}{\alpha}\left(\frac{q_{i}}{o_{i}}-\frac{g_{i}}{p_{i}}\right)^{2}
$$

where $o_{i}$ is the amount of automated information processing on the $i$-th intelligent node; $p_{i}$ is the total amount of information processing on the $i$-th intelligent node; $q_{i}$ is the information automation of the $i$-th intelligent node designed for the entire green environment importance; $g_{i}$ is the evaluation value of the single-period shared data of the $i$-th smart node; $\alpha$ is the total number of rows in the grid; $\beta$ is the total number of columns in the grid.

Scene production based on virtual reality technology can be combined and designed by constructing different elements such as buildings, roads, lawns, terrain, plants, and sky. According to the principle of ergonomics, the three elements of human, machine, and environment are organically unified to achieve the effect of full-scene, realperson visual roaming [10]. In ergonomics, it is necessary to analyze the characteristics of human body height and eye height, differences, and regional characteristics.

\section{AI-Based Technical Framework of Green Environmental Design}

3.1. Program Design. The general method to realize particle swarm optimization is to establish an operational model of the design task; the establishment of the model includes two stages. First, cognitive modeling, that is, the data-driven concept learning stage, is used to form customization and form a reasonable spatial layout. From the beginning of its birth, green environment design has been based on the design methods of mathematics and geometry, the use of materials, and engineering. As a drawing tool and visualization method, computer tools have played a huge auxiliary role in the modern environmental design industry, but they cannot ultimately affect the designer's decision-making process. With the advent of computers, the engineering means and design methods provided by computers are a fundamental change in modern environmental art, especially architectural design [11]. In the field of green environmental design, the design system of environmental art is a complex and multidimensional cross-domain fusion system, which can be regarded as a large task system. Although the application of particle swarm optimization in the partial aspects of environmental design has been successful, such as big data collection, virtual reality, architectural structure, and style analysis, interior furnishings and style analysis still need to be improved.

The collaborative AI system is a new intelligent system that integrates multiple core technologies such as computer voice, image recognition, deep learning, natural language processing, and design atlas. The driving effect of AI on green environment design innovation is mainly reflected in the process automation and the intelligence of management 
itself, including the intelligence of data collection, feedback, monitoring, evaluation, analysis and prediction, and decision-making. It promotes the evolution of the weak matrix structure of the organization to the strong matrix structure, forming an organizational structure that focuses on the improvement of organizational innovation capabilities vertically and AI technology and green environment design horizontally (Figure 1). This structure not only simply breaks the original pattern and breaks some inherent boundaries and responsibilities when optimizing the design, but also changes the configuration and organization of the design. In Figure 1, on the one hand, it designs a series of parts and components according to the general design method, from which the ideas and content of green design must be reflected; on the other hand, it uses network technology to realize the compatibility and overall management of the original information system of the production enterprise and establishes a green design as the databases of main content. This kind of flexibility in intelligent capabilities can ensure that organizations can effectively weigh flexibility and efficiency, quickly respond to uncertain changes, and gain a competitive advantage [12].

AI interconnection technology realizes the intelligent interconnection of people and things. Green environment layout, resources, databases, designers, four types of management intelligent collaboration, and in-depth mining of internal and external data resources all rely on the depth of AI learning mode. Furthermore, an intelligent service model driven by demand data and intensive information is formed. $\mathrm{AI}$ is an assistant to promote the design of green environment, providing more comprehensive support in terms of basic technology. AI extends the green environment design information collection methods, and virtual sensing technologies increase the channels of information sources, and the dimensions of obtaining information continue to extend. The methods of processing information must comprehensively use AI technology to adapt to the logic of information services. It first comprehensively collects and deeply integrates the green environment design literature database, historical data, and designer information and then develops a green environment design innovation platform based on AI technology to build a complete, professional, and accurate foundation. The ant colony optimization comprehensively enhances the breadth and depth of green environmental design and improves efficiency and scope of influence; it collects, processes, utilizes, and feeds back data in real time during human-computer interaction, realizing group information sharing and promoting automatic information migration.

3.2. Function Development. Distributed AI is a new AI research field that is based on technologies such as computer networks, computer communications, and concurrent programming to simulate the way that human thinking solves large and complex problems in a social environment. Distributed intelligence refers to intelligent systems or intelligent objects that are logically or physically distributed in solving large-scale complex problems, the intelligence displayed by coordinating their respective intelligent behaviors [13]. The application of genetic algorithm in green environment design mainly reflects the intelligent processing of behaviors and actions. For example, according to the recognized actions, the green environment structure is activated differently. After the sensor completes the information collection, it will routinely integrate the information and make activation judgments. Therefore, it is assumed that the main detection of this research is the distance between people and obstacles in a green environment or the motion information related to obstacles, and the output of the rotation angle changes and speed changes of people in the green environment are output (Figure 2). As a new type of intelligent algorithm, evolutionary control algorithm is mainly composed of traditional evolutionary computing theory and feedback control theory, which has important value for improving adaptability and learning ability in green environment design.

The existing AI automated design system can only massproduce content such as title pictures for interface homepages or pop-up windows and small-format electronic posters or single page layouts of presentation documents; and the applicable strategies of products are all based on the high-speed iterative method of constant replacement in the main. In Figure 2, the development and application of AI technology can effectively solve many problems in the design of green environment. For example, the technical problems involved in the green environment design process are difficult to solve efficiently for technicians with insufficient knowledge reserves. At the same time, when designing a green environment, the application of AI technology can also help people integrate into the environment. Rules usually appear in the form of design templates in the AI design process. Their function is to restrict the layout of elements after the elements are classified, so as to form alternatives that initially meet the design criteria and visual requirements. At present, the template composition rule used in AI design is to lay out the relative positional relationship and space occupation of different types of elements. AI uses the graphic and image information of the elements to simulate the artificial process to combine, and it is oriented to complex semantics determined by the adaptive characteristics of the environment.

The application of AI technology in the design of green environment can realize the extraction and analysis of the salient features of the object through the feature extraction technology. After the extraction is completed, the particle swarm optimization will rely on its own advantages to compare and match the collected feature information with the information in the pattern library. In other words, this link needs to realize the reasonable use of pattern matching technology to ensure that AI technology can give full play to its value and achieve the purpose of green environmental design. Using AI technology as the basis, the operation thresholds corresponding to pattern matching can be compared in the link of green environment design. For example, the similarity is used as a measure of pattern matching, and a corresponding threshold is set; after the threshold is set, the characteristic information of the object 

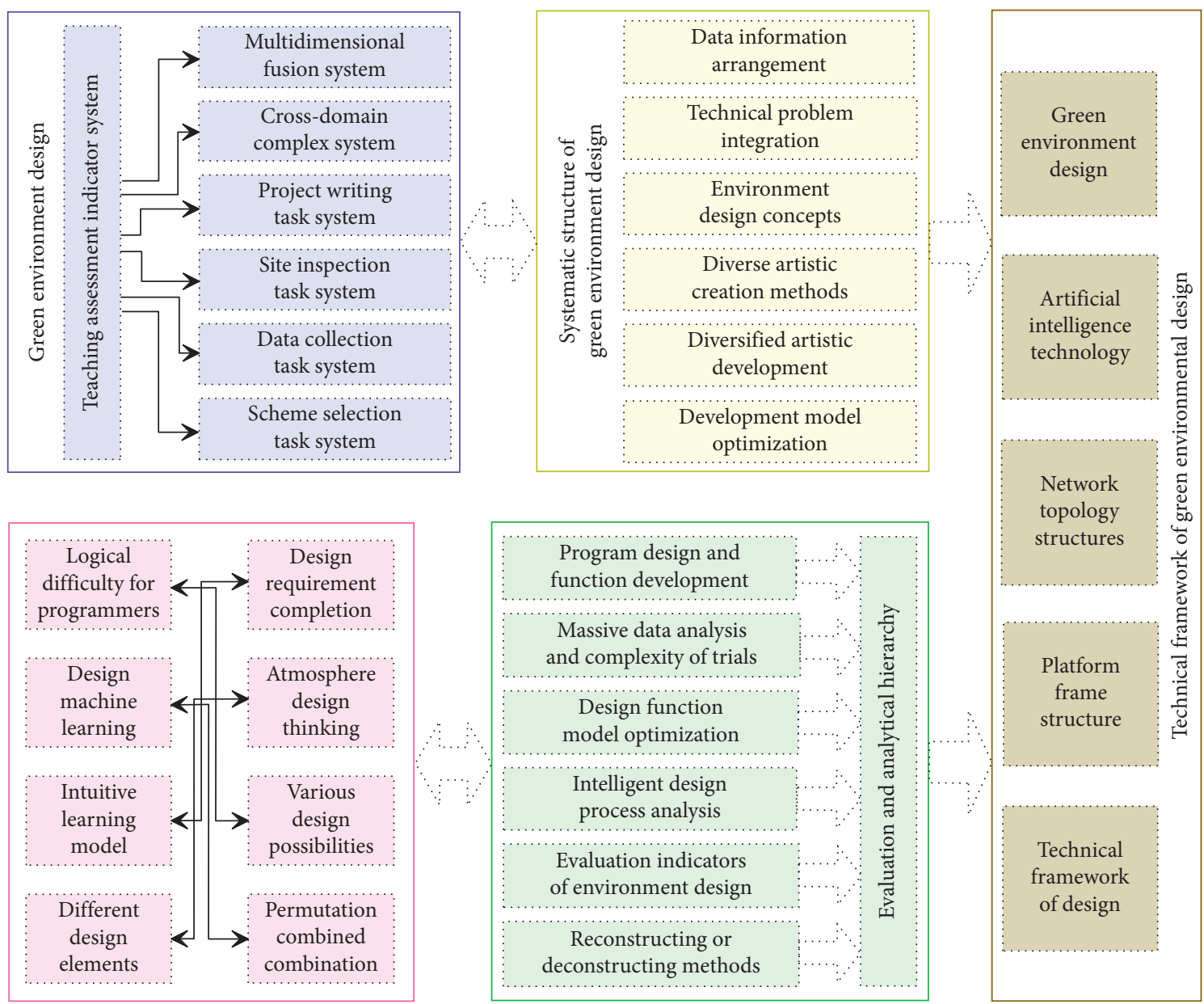

FIgURE 1: Analytical framework of application mode of green environment design under the AI background.

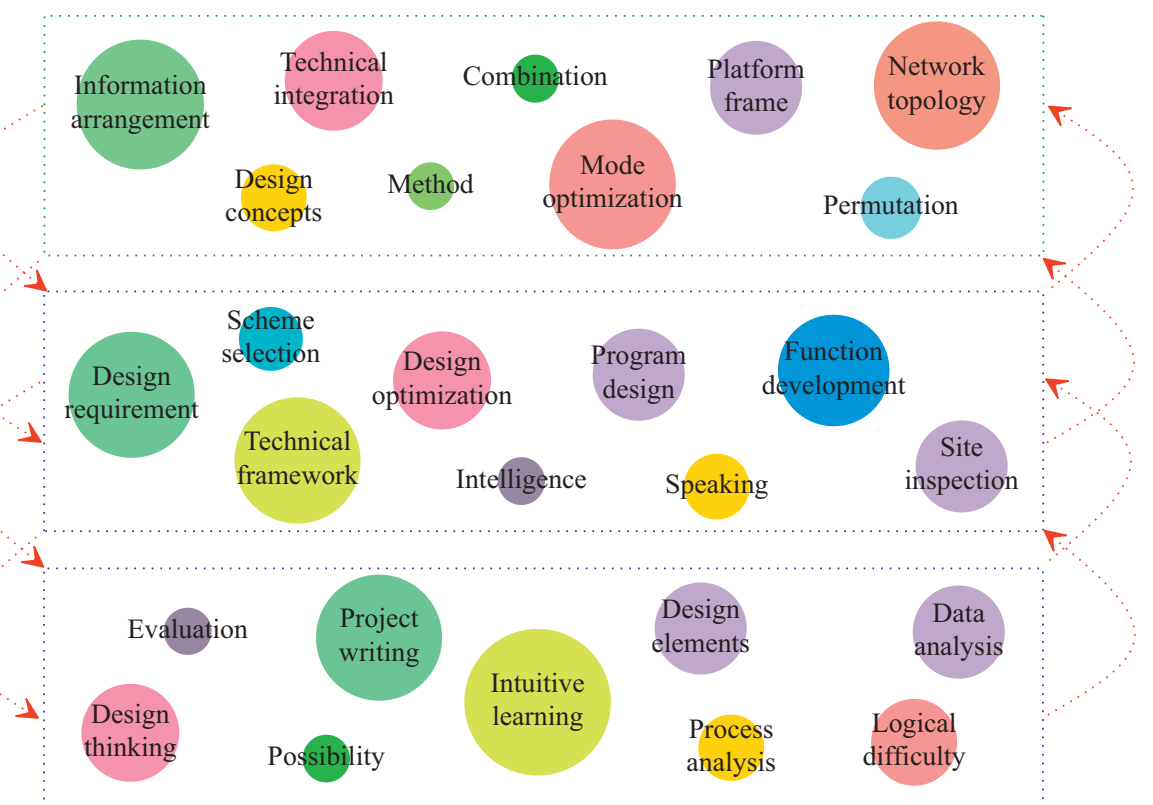

FIGURE 2: Technical framework of program design and function development in green environmental design under the AI background. 
can be compared [14]. Assuming that the similarity is found to be higher than the set threshold, it means that the matching result is successful this time. On the contrary, it is a failure and it is necessary to check and analyze the threshold setting, feature extraction, and other links to determine whether there is an abnormal phenomenon. When the matching link of the green environment design is completed and the matching result is successful, the AI technology will convert the machine language accordingly, so that it is presented in a natural language that people can recognize and understand.

\section{Application Mode of Green Environment Design under the AI Background}

4.1. Functional Model. In view of the new developments and hotspots of green environmental design, the new theories and new methods of AI theory in the field of design information mining are incorporated into the practical links of environmental design in a timely manner, which can not only broaden the designer's learning horizons, but also exercise practical skills. In design practice, it not only involves knowledge of previous technologies such as program design, data structure, probability, and statistics, but also involves the latest AI technologies and methods, which are highly comprehensive. How to rationally lay out basic and cutting-edge knowledge, deeply integrate theory and practical application, and at the same time improve the designer's sense of accomplishment in system design and implementation is the key to motivating designers to explore and be creative. Green environmental design involving goals, resources, and behaviors can be dealt with from an economic perspective, using intelligent mechanism models to reflect the development trend of models from centralized to decentralized control and behavior. The intelligent design mechanism can integrate local decision-making into a global effective behavior, showing its important value in the organization of large-scale distributed systems. The second one is the smart control strategy, which captures the parallel characteristics of the manufacturing system, that is, the execution of each process in the environment design and the completion of functions are simultaneous, and centralized scheduling coordination is necessary [15].

The framework correctly and clearly conducts system analysis to form a standardized design document system, helps to correctly formulate system function division and software module design strategies, and helps software development organizations to systemize and recycle software components. If designer only relies on the fixed style interface of the existing expert system development platform, it will greatly affect the support efficiency of intelligent decision-making, and users will also use it (Figure 3). The inference engine is initialized inside the controller, and then the inference engine is started, and the matching result is returned to the controller as the updated information of the model function. The current known data is stored in the working memory, and these data are activated by the rule interpreter to activate the corresponding rules in the production rule library [16]. In Figure 3, the element probability
$Q(a, b, c)$ is based on green plants and public facilities, while the artificially intelligent green environment can realize resource sharing. The matrix similarity $f(a, b, c)$ complements each other with local humanities and realizes the organic combination of multiple benefits. The elevation scale $\left|R_{x}-T_{x}\right|$ breaks away from the traditional interface-style interactive thinking and integrates the research methods of emotional computing. The intelligence index $O(x)$ proposes three interactive design methods, differentiated design for subdivided groups, product active intelligent interactive design, and virtual reality scene interactive design, and explains how to apply AI technology in interactive design. According to the principles of green environment design, and then associating the knowledge base structure with the database, it can be found that the AI system is a knowledge system represented by rules, where the knowledge base is the source of the function of the expert system, and the rules are the specific application of the knowledge of the expert system.

AI technology uses computer technology and information technology as the means to help middle- and highlevel designers carry out design activities on semistructured and unstructured green environment design issues. The mode provides designers with the data, information, and materials needed for design and helps designers clarify the design goal and the understanding of the problem, establishes and modifies the design model, provides various selection plans, and optimizes, analyzes, compares, and judges the various strength plans to help the designer improve the design ability, level, quality, and benefits in order to achieve the greatest economic and social benefits. As long as the system integration in modern intelligent buildings is established to meet the needs of building internal service automation, it is a technical means from traditional automation systems to modern management development. AI technology can build a powerful sample size for nonlinear data mining, which can be more effectively applied to highlatitude data patterns. The most critical objective function of the support vector machine is the kernel function. If the sample is in a low-dimensional space, the solution is to map it to a high-dimensional space, but it is easy to increase the computational complexity. When applying, choose a suitable kernel function and map it to a high-dimensional space for classification, so more objective functions can be obtained.

4.2. Process Analysis. From the perspective of the evolution of technical methods, green environmental design has emerged in the development process of etiquette-oriented, aesthetic-oriented, modernism, social-oriented, ecologicaloriented, and digital green environmental design and other thematic features. As an important part of the green environment design, it is mainly to inject ecological theory and analysis methods on the basis of traditional green environment design to achieve the planning goal of sustainable urban development. On the whole, ecologically-oriented green environment design seeks to construct a human settlement space that is coordinated with the artificial 


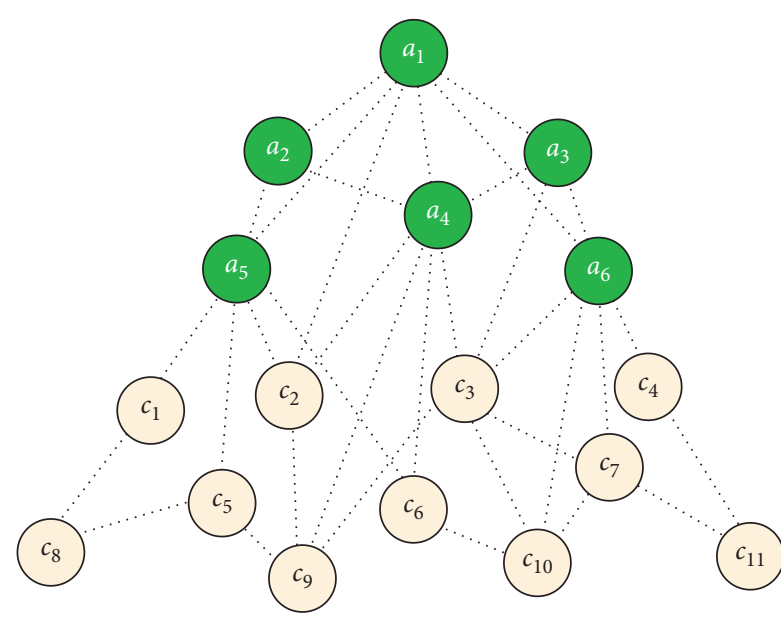

(a)

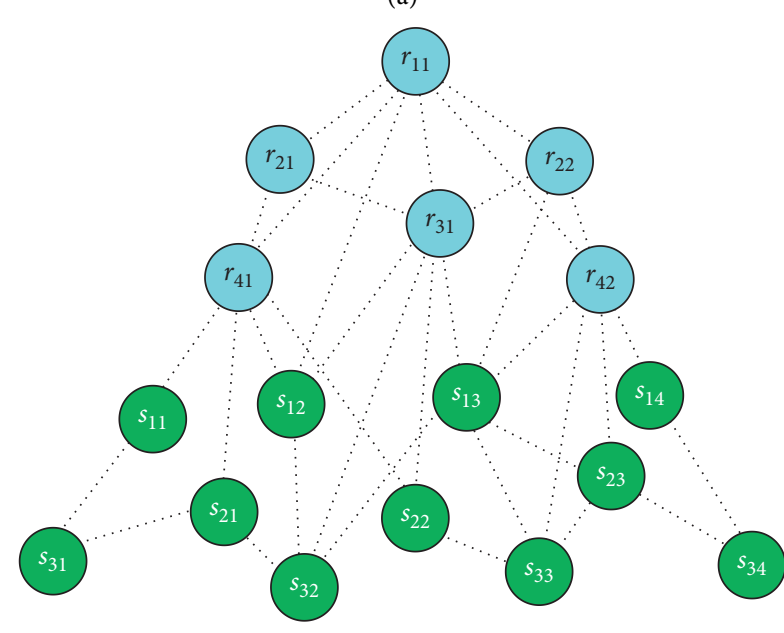

(c)

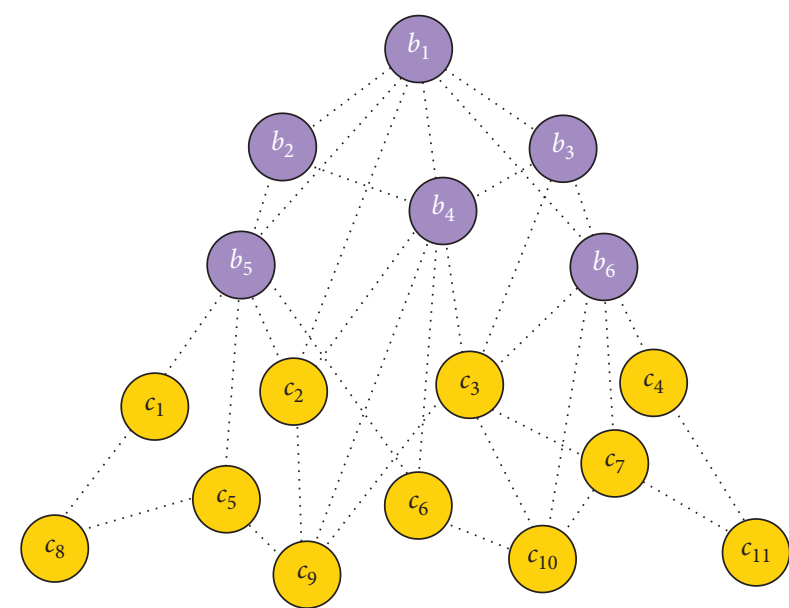

(b)



(d)

FIGURE 3: Element probability (a), matrix similarity (b), evaluation scale (c), and intelligence index in the green environment design under the AI background.

environment and the natural environment. It has a significant practical guiding role for the sustainable development of urban environmental construction and can effectively improve the quality of the urban ecological environment (Figure 4). They improve land use efficiency, beautify urban spatial landscape, and improve energy efficiency, and the foundation of AI design is data. The collection, screening, cleaning, processing, analysis, and application of massive data are a full path for the application of technical methods. To deal with diversity, the analysis and utilization of multidimensional data still need to be explored, and the overall analysis level needs to be improved. The penetration of digital technology into traditional technology and methods is fast or slow, but the architecture of AI design methods is relatively complete.

Green environment design integrates knowledge from multiple domains, including aesthetic expressions and logical thinking in philosophical concepts. For example, intelligent computer graphics can be designed as a series of mathematical modeling tasks, in which cognitive science and AI provide a concept library for describing and designing calculation models and then automatic drawing can be realized based on the calculation models [17]. This model can combine the development status of AI and green environment design tools, analyze the combination of $\mathrm{AI}$ and green environment design and future development trends, and give some empirical applications of using AI for green environment design. AI provides designers with new design integration points, gradually extending traditional fixed artistic creation concepts and producing more diverse artistic creation methods and gradually shifting from fixed models to more diversified artistic thinking (Figure 5). The ant colony optimization has injected new blood into green environmental design, enabling designers to have a new understanding of artistic creation and generating new design concepts, making green environmental design bloom new vitality under the integration of science and technology.

Vector objects describe spatial objects through the combination of geographic information and object attributes; vector data files can be converted into binary or text format for further subsequent processing by the designer. The application of AI technology to green environment 


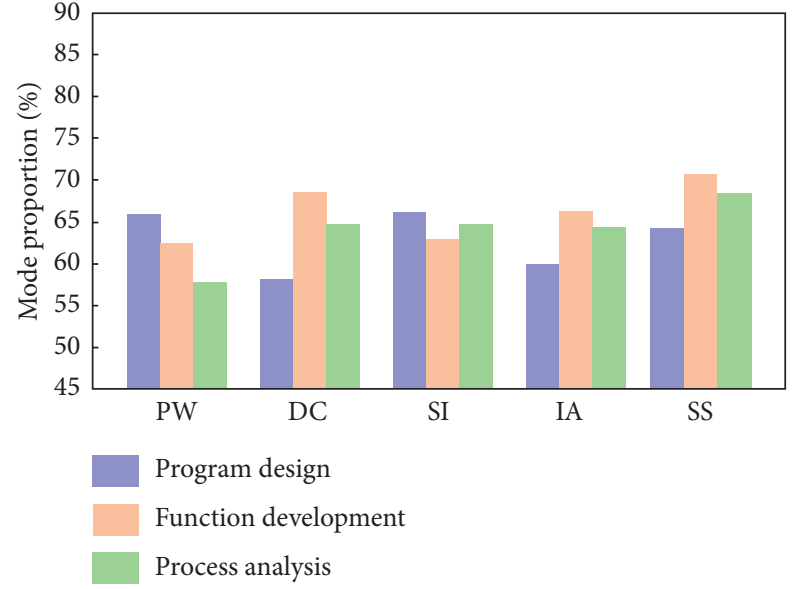

(a)

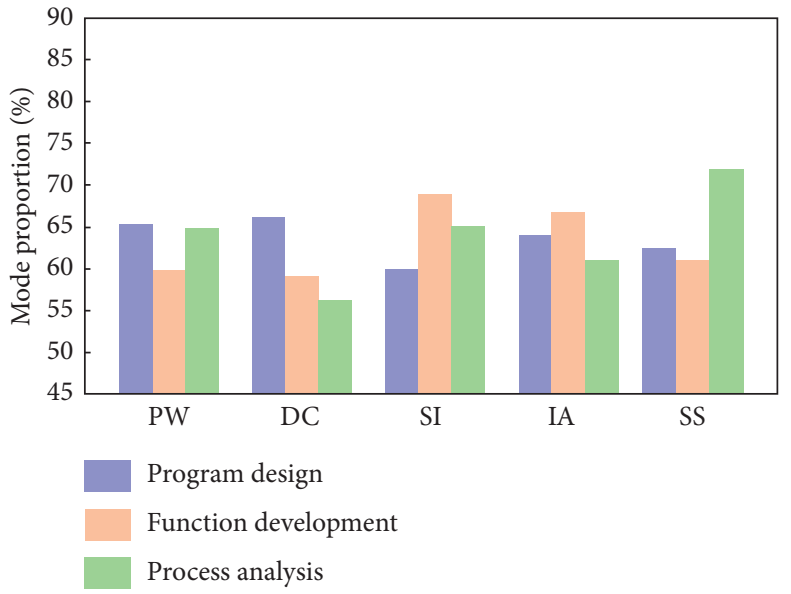

(b)

Figure 4: Mode proportion of five different green environment design dimensions in the functional model (a) and hierarchical analysis (b) under the AI background. Note: PW: project writing; DC: data collection; SI: site inspection; IA: information arrangement; SS: scheme selection.

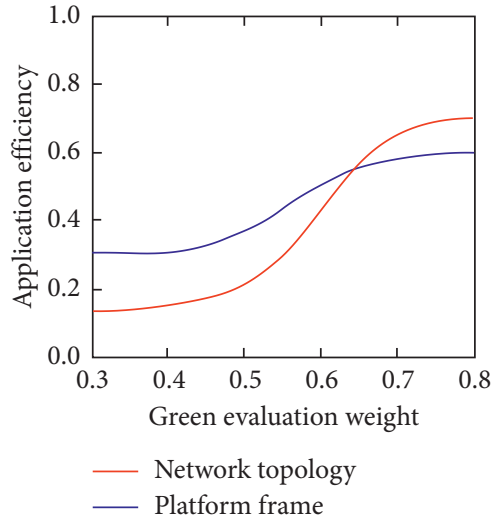

(a)

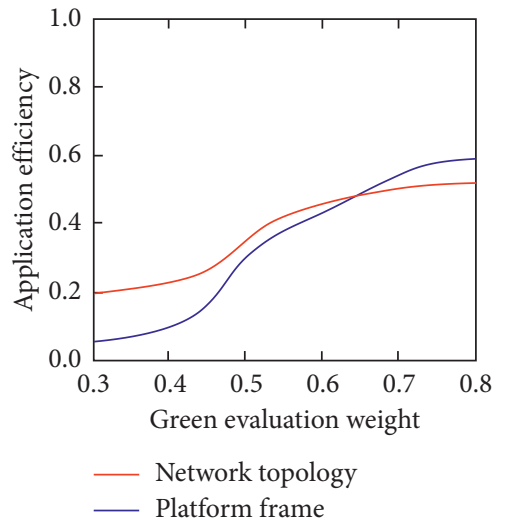

(d)

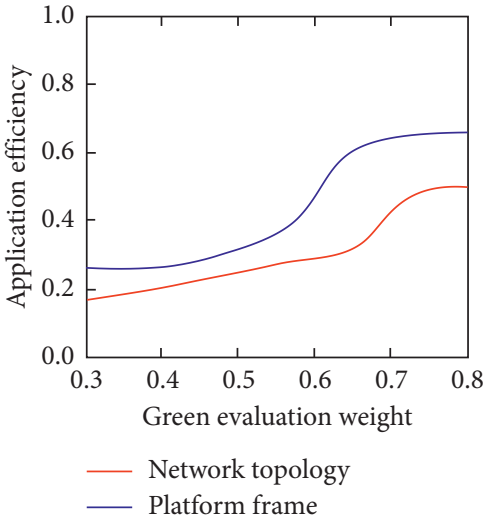

(b)

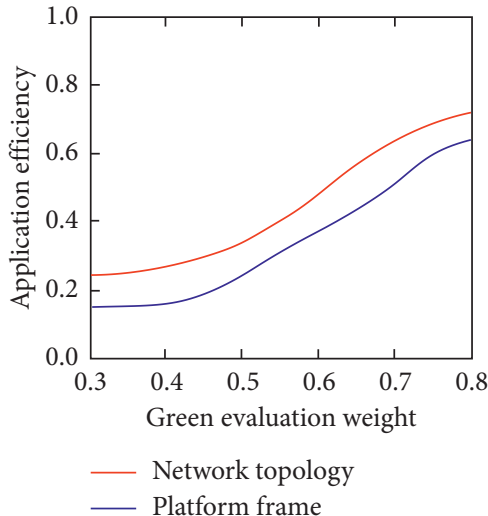

(e)

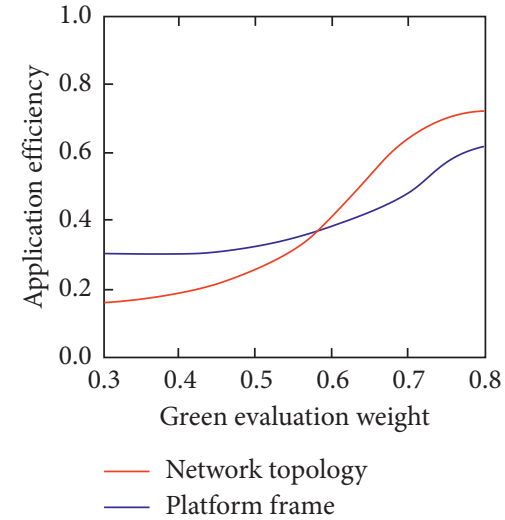

(c)

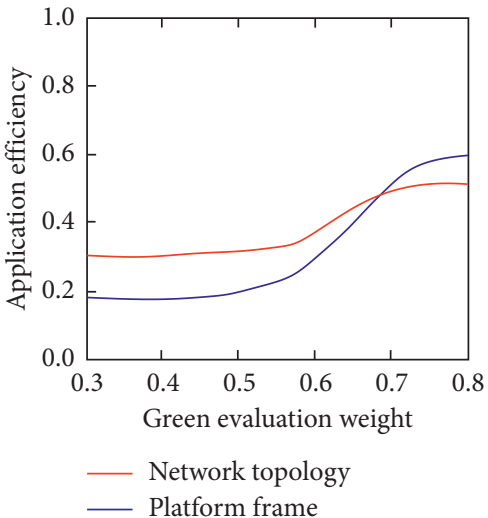

(f)

Figure 5: Application efficiency of green environment design in network topology and platform farm under the AI background.

design can simplify calculation complexity, facilitate data mining and utilization, obtain commercial and academic value from massive data, and improve the processing efficiency of green environment design data [18]. However, compared with AI in the general industry, not only does green environment design have a huge amount of data, but 
its complex data organization and diverse data structure also bring considerable challenges to design management. In information systems, many management tasks usually require the use of multiple types of heterogeneous data for calculation and analysis, and its data format is difficult to be consistent. A system that can provide public management services needs to deal with a large amount of heterogeneous and irregular design data. Real-time response to data processing requests submitted by different design management tasks is an important task.

\section{Simulation Experiment and Analysis}

5.1. Experimental Design. Based on the close integration of AI technology with green environmental design, it will give full play to its advanced technology, which will surely bring huge benefits to the economy and society. According to statistics, it has achieved certain success in various professional fields such as environmental structure and construction, environment and design, and project management. With the rapid development of network technology, big data, and computer computing capabilities, based on the opening of the big data era, especially with the continuous maturity of database technology, the introduction of intelligent decision-making systems in the integration of environmental intelligent systems can make the intelligent environment truly realized networks [19]. Green and intelligent environmental design is based on the scientific knowledge and understanding of the natural ecology to create an ecologically friendly environment space, and the method of constructing environmental design is derived from the many mysterious functions of nature. In order to realize the role of green $\mathrm{AI}$ in environmental design, the cutting-edge technology and environmental protection design of green intelligent environmental design can also construct a cutting-edge large database system. It needs to consider the design method and realization path of a reliable environment that is close to nature, while achieving construction, maintenance management, and abandonment, saving energy and resources, and pursuing the organic connection between environmental design and the natural environment (Figure 6).

In Figure 6, in the network topology structure, when the node probability ranges from 0.1 to 0.7 , most of the matrix similarity is distributed between -0.2 and 0.1 ; in the platform frame structure, when the node probability ranges from 0.1 to 0.7 , most of the matrix similarity is distributed between -0.1 and 0.2 . Since the diversity of green design has rich sociological connotations, it is not only the diversity of environmental functions, but also the diversity of species and the diversity of human activities. The main advantages of green materials are that they can save resource consumption, improve resource utilization, and reduce the impact on the natural ecological environment [20]. At the same time, green materials are more outstanding in function and can reflect the problems of recycling and treatment that traditional materials cannot achieve. The environmentally friendly attributes of the company meet the core requirements of the green design concept to the greatest extent. This is an excellent way to practice and apply the green concept and use the laws of nature to design, so as to organically combine the environmental art design and the green ecosystem, and think about the style, shape, color, material, scale, function, and layout of the building and the environment from the overall relationship.

5.2. Result Analysis. AI technology can not only make green environment design more inclusive, but also sacrifice minimum performance to reduce computing overhead and gradually improve performance as related technology advances. The role of particle swarm optimization in green environment design also includes the application of waterscape design. As an important part of the green environment, waterscape design is the core of the entire environmental ecosystem. With the development and popularization of AI technology, designers have gradually introduced intelligent sensing systems into environmental design (Figure 7). The waterscape landscape systems such as music fountains, water purification systems, and intelligent rainwater systems have begun to enter the sight of the people. It complements the current recyclable ideas and has significant effects. With the help of technology, the intelligent system can perceive the surrounding environment of the green environment and adjust the basic attributes such as the intensity, color, and direction of the light according to the actual situation, making it an intelligent device that can interact with surrounding characters and scenery, and enhance the value of the landscape [21]. The combination of music and landscape is also an important direction for the development of AI technology. Music is used to cleanse the soul, combine people with music, and enhance the atmosphere of the landscape.

When a designer puts design thinking into design behavior, it is a process of constant trial and error. For people, the complexity of trial and error will directly affect the designer's final plan, because people's knowledge, experience, and energy are limited. AI learning design thinking relies on massive data analysis, that is, finding the commonalities of things and marking them as samples for archiving. When the design and production process is started, the AI design platform will combine the needs of customers according to the laws it has mastered. This includes reconstructing or deconstructing methods, carrying out various possibilities of trial and error, and relying on super fast advantages to complete the design requirements. Therefore, AI design is trying to flatten design thinking and human design thinking is by no means flat. It puts all the design factors that need to be considered on a plane and obtains various design possibilities through permutation and combination, which will produce a large number of similar design results (Figure 8). This method of flattening design thinking is to reduce the logical difficulty for programmers to design machine learning and to construct a more intuitive machine learning model. In the same design goal direction, because it subjectively will pursue the differentiated performance of the use of various design elements, it can produce more different design results. 


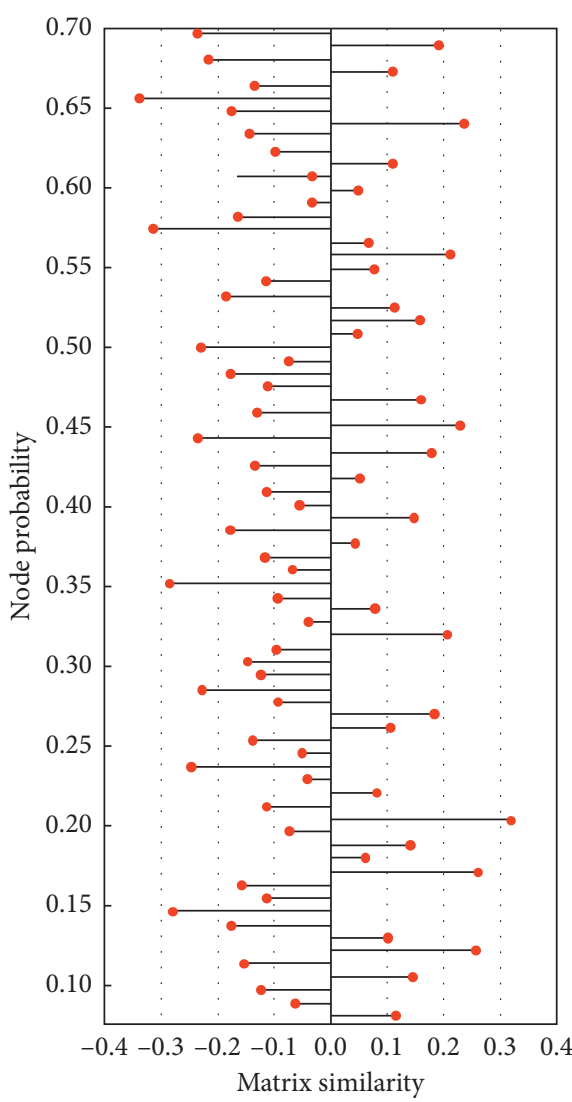

(a)

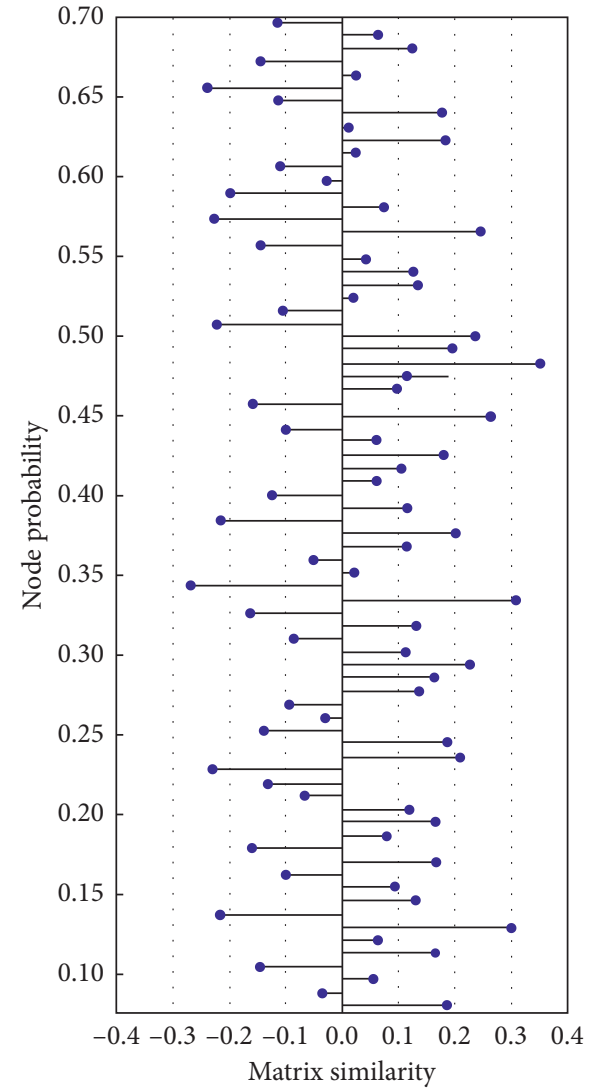

(b)

FIGURE 6: Distribution of node probability with different matrix similarities in the network topology structure (a) and platform frame structure (b).

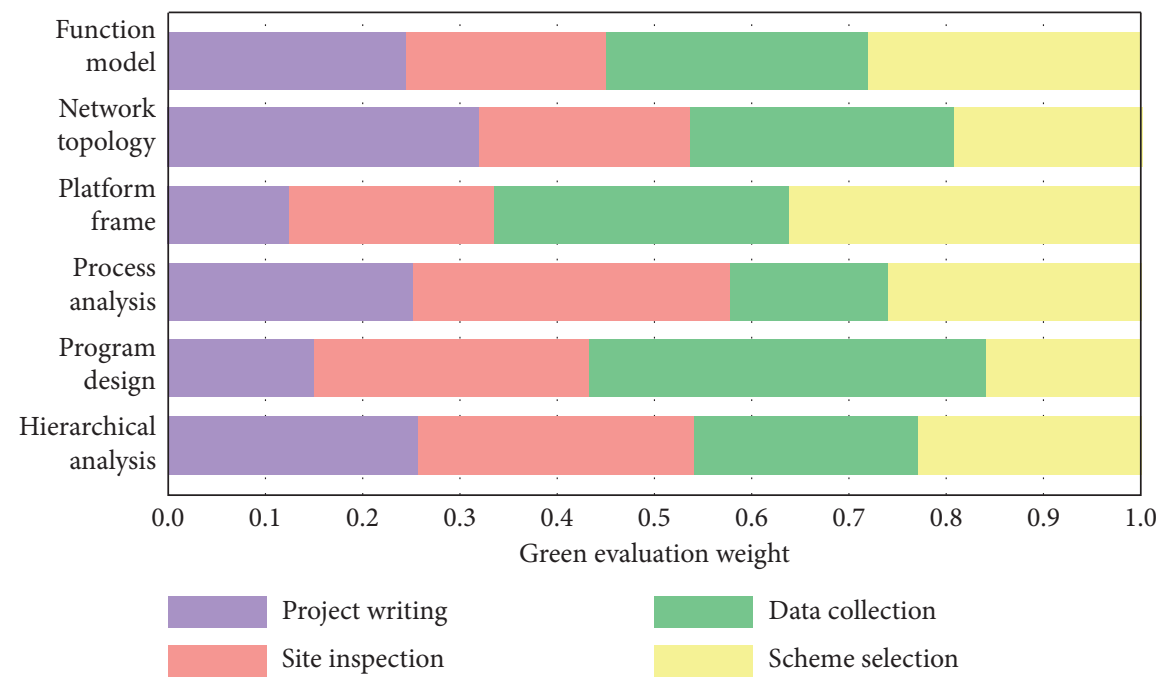

FIGURE 7: Distribution of green evaluation weight in green environment design under the AI background.

Green environmental design is a complex design system. Research on green environmental design requires the combined use of a variety of professional knowledge, comprehensive analysis, and comparison. Each of the above stages may have an indirect or direct impact on the environment, and the designer's past experience and subjective decision-making ability alone cannot adapt to the requirements of complex designs. The complete green environmental design starts from the analysis of the environment in the preliminary design and finally reaches the 

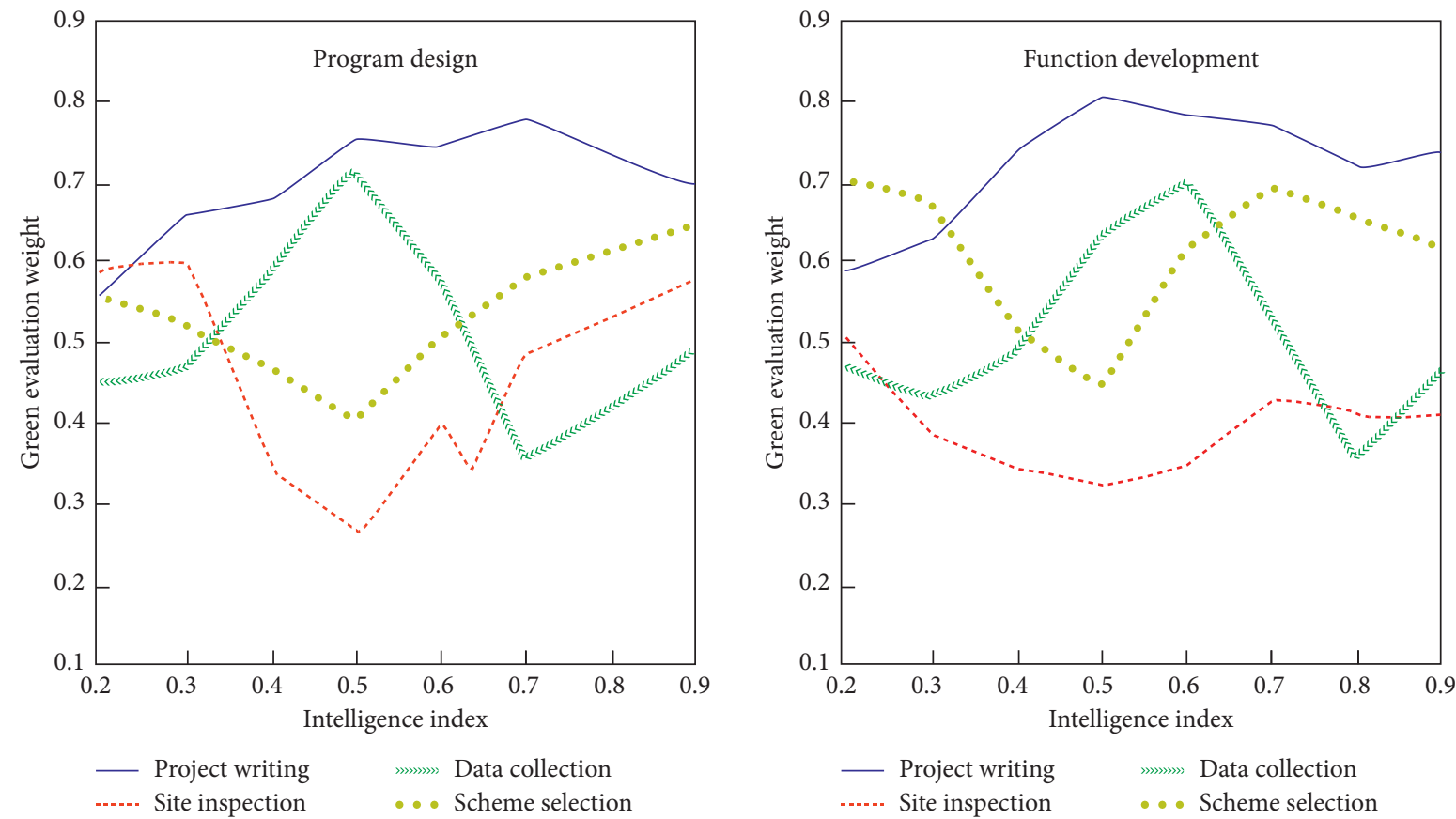

FIGURE 8: Relationship between green evaluation weight and intelligence in program design and function development under the AI background.

requirements of a circular design after in-depth design, construction, use, and recycling. The construction of the green environmental design evaluation indicators system is to put forward a reasonable analysis and evaluation of the pollution and impact that each link in the environmental design may have on the environment and screen out the most reasonable use of resources, the least energy consumption, and the environmental pollution damage caused by the design process and manufacturing process [22-33]. The best green environment design plan can obtain more economic benefits and can have more sustainable and longterm development. Research on green environment design is conducive to establishing people's green design concepts, and it is conducive to designers to use multiangle scientific analysis of each link in the design and analyze the amount of pollution and impact that the design environment will have on the environment during the entire service life of the design environment. The main reason is to find more advanced technical means in environmental design, as well as more reasonable ways to utilize resources and energy.

\section{Conclusions}

This paper introduced the network topology and platform frame structures of green environment design under the AI background, constructed a technical framework of green environment design under the AI background, analyzed the methods and principles of program design and function development, proposed the application model of green environment design under the AI background, conducted design function model optimization and intelligent design process analysis, and finally discussed the evaluation indicators and hierarchical analysis of the green environment design under the AI background. Green environmental design is a complex design system and research on green environmental design requires the combined use of a variety of professional knowledge, comprehensive analysis, and comparison. On the whole, ecologically-oriented green environment design seeks to construct a human settlement space that is coordinated with the artificial environment and the natural environment. AI technology can not only make green environment design more inclusive, but also sacrifice performance to reduce computing overhead and gradually improve performance as related technology advances. AIdriven design is one of the issues being discussed in the art world and it can help designers get rid of tedious design steps in some aspects, save design time, and improve efficiency. The results show that the application mode of combining AI technology and green environment design can not only help designers break through traditional time and space barriers and use multidimensional thinking, but also help designers have a new understanding of artistic design concept and give full play to the advantages of artificial intelligence in a green environment. The study results of this paper provide a reference for further researches on the application mode of green environment design under the AI background. Further researches should keep analyzing the idea that green environment layout, resources, databases, designers, four types of management intelligent collaboration, and in-depth mining of internal and external data resources all rely on the depth of AI learning mode.

\section{Data Availability}

Data sharing is not applicable to this article as no datasets were generated or analyzed during the current study. 


\section{Conflicts of Interest}

The authors declare that there are no conflicts of interest.

\section{Acknowledgments}

This project was supported by the Program of Henan Provincial Department of Education: Research on Green Design Strategy of Transforming Traditional Dwellings into Homestay under Rural Revitalization Strategy (2021-ZZJH132); Study on the Design PATH of the Integration of Chinese and Tourism in Jiaozuo under the Background of Global Tourism (THZK202012); and Research on Ecological Design of Traditional Residential Buildings in Southern Henan (Henan Polytechnic University's 2016 Youth Backbone Teacher Funding Project).

\section{References}

[1] A. Fallahpour, E. U. Olugu, S. N. Musa, D. Khezrimotlagh, and K. Y. Wong, "An integrated model for green supplier selection under fuzzy environment: application of data envelopment analysis and genetic programming approach," Neural Computing and Applications, vol. 27, no. 3, pp. 707725, 2016.

[2] M. F. Hossain, "Green science: advanced building design technology to mitigate energy and environment," Renewable and Sustainable Energy Reviews, vol. 81, pp. 3051-3060, 2018.

[3] S. Maruthaveeran, "The perception of social safety in a green environment: a preliminary study at the Kepong Metropolitan Park," Asian Journal of Environment-Behaviour Studies, vol. 1, no. 1, pp. 99-111, 2016.

[4] M. Jaganmohan, S. Knapp, C. M. Buchmann, and N. Schwarz, "The bigger, the better? The influence of urban green space design on cooling effects for residential areas," Journal of Environmental Quality, vol. 45, no. 1, pp. 134-145, 2016.

[5] S. Gupta, U. Soni, and G. Kumar, "Green supplier selection using multi-criterion decision making under fuzzy environment: a case study in automotive industry," Computers \& Industrial Engineering, vol. 136, pp. 663-680, 2019.

[6] W. Zhu and Y. He, "Green product design in supply chains under competition," European Journal of Operational Research, vol. 258, no. 1, pp. 165-180, 2017.

[7] G. Tian, H. Zhang, M. Zhou, and Z. Li, "AHP, gray correlation, and TOPSIS combined approach to green performance evaluation of design alternatives," IEEE Transactions on Systems, Man, and Cybernetics: Systems, vol. 48, no. 7, pp. 1093-1105, 2017.

[8] B. Shen, Y. Cao, and X. Xu, "Product line design and quality differentiation for green and non-green products in a supply chain," International Journal of Production Research, vol. 58, no. 1, pp. 148-164, 2020.

[9] B.-H. Li, B.-C. Hou, W.-T. Yu, X.-B. Lu, and C.-0. Yang, "Applications of artificial intelligence in intelligent manufacturing: a review," Frontiers of Information Technology \& Electronic Engineering, vol. 18, no. 1, pp. 86-96, 2017.

[10] R. Li, Z. Zhao, X. Zhou et al., "Intelligent 5G: when cellular networks meet artificial intelligence," IEEE Wireless Communications, vol. 24, no. 5, pp. 175-183, 2017.

[11] M. Perez-Valls, J. Cespedes-Lorente, and J. Moreno-Garcia, "Green practices and organizational design as sources of strategic flexibility and performance," Business Strategy and the Environment, vol. 25, no. 8, pp. 529-544, 2016.
[12] J. Heer, "Agency plus automation: designing artificial intelligence into interactive systems," Proceedings of the National Academy of Sciences, vol. 116, no. 6, pp. 1844-1850, 2019.

[13] B. Pirouz, M. Turco, and S. A. Palermo, "A novel idea for improving the efficiency of green walls in urban environment (an innovative design and technique)," Water, vol. 12, no. 12, p. 3524, 2020.

[14] X. Yang, Y. Wang, R. Byrne, G. Schneider, and S. Yang, "Concepts of artificial intelligence for computer-assisted drug discovery," Chemical Reviews, vol. 119, no. 18, pp. 10520-10594, 2019.

[15] T. Snäll, J. Lehtomäki, A. Arponen, J. Elith, and A. Moilanen, "Green infrastructure design based on spatial conservation prioritization and modeling of biodiversity features and ecosystem services," Environmental Management, vol. 57, no. 2, pp. 251-256, 2016.

[16] A. Rezaee, F. Dehghanian, B. Fahimnia, and B. Beamon, "Green supply chain network design with stochastic demand and carbon price," Annals of Operations Research, vol. 250, no. 2, pp. 463-485, 2017.

[17] R. Rahinel and N. M. Nelson, "When brand logos describe the environment: design instability and the utility of safety-oriented products," Journal of Consumer Research, vol. 43, no. 3, pp. 478-496, 2016.

[18] T. S. Khan, F. Jalid, and M. A. Haider, "First-principle microkinetic modeling of ethanol dehydrogenation on metal catalyst surfaces in non-oxidative environment: design of bimetallic alloys," Topics in Catalysis, vol. 61, no. 18-19, pp. 1820-1831, 2018.

[19] A. S. Klymchenko, "Solvatochromic and fluorogenic dyes as environment-sensitive probes: design and biological applications," Accounts of Chemical Research, vol. 50, no. 2, pp. 366-375, 2017.

[20] T. Mäkelä, S. Helfenstein, M.-K. Lerkkanen, and A.-M. Poikkeus, "Student participation in learning environment improvement: analysis of a co-design project in a Finnish upper secondary school," Learning Environments Research, vol. 21, no. 1, pp. 19-41, 2018.

[21] A. K. Das, M. Wazid, N. Kumar, M. K. Khan, K. K. R. Choo, and Y. Park, "Design of secure and lightweight authentication protocol for wearable devices environment," IEEE Journal of Biomedical and Health Informatics, voll, vol. 22, no. 4, pp. 1310-1322, 2017.

[22] D. T. Tiruneh, A. G. Weldeslassie, A. Kassa, Z. Tefera, M. De Cock, and J. Elen, "Systematic design of a learning environment for domain-specific and domain-general critical thinking skills," Educational Technology Research and Development, vol. 64, no. 3, pp. 481-505, 2016.

[23] L. Ding, S. Li, H. Gao, H. Chen, and Z. Deng, "Adaptive partial reinforcement learning neural network-based tracking control for wheeled mobile robotic systems," IEEE Transactions on Systems, Man, and Cybernetics: Systems, vol. 50, no. 7, pp. 2512-2523, 2018.

[24] Y. Chen, W. Zheng, W. Li, and Y. Huang, "Large group Activity security risk assessment and risk early warning based on random forest algorithm," Pattern Recognition Letters, vol. 144, pp. 1-5, 2021.

[25] J. Wen, J. Yang, B. Jiang, H. Song, and H. Wang, "Big data driven marine environment information forecasting: a time series prediction network," IEEE Transactions on Fuzzy Systems, vol. 29, no. 1, pp. 4-18, 2021.

[26] W. Wei, M. Guizani, S. H. Ahmed, and C. Zhu, "Guest editorial: special section on integration of big data and artificial 
intelligence for Internet of Things," IEEE Transactions on Industrial Informatics, vol. 16, no. 4, pp. 2562-2565, 2020.

[27] J. Qian, X. Cheng, B. Yang et al., "Vision-based contactless pose estimation for human thermal discomfort," Atmosphere, vol. 11 , no. 4 , p. 376, 2020.

[28] W. Wang, Z. Gong, J. Ren, F. Xia, Z. Lv, and W. Wei, "Venue topic model-enhanced joint graph modelling for citation recommendation in scholarly big data," ACM Transactions on Asian and Low-Resource Language Information Processing (TALLIP), vol. 20, no. 1, pp. 1-15, 2020.

[29] A. Larkin and P. Hystad, "Evaluating street view exposure measures of visible green space for health research," Journal of Exposure Science \& Environmental Epidemiology, vol. 29, no. 4, pp. 447-456, 2019.

[30] E. C. A. Nordbø, R. K. Raanaas, H. Nordh, and G. Aamodt, "Neighborhood green spaces, facilities and population density as predictors of activity participation among 8-year-olds: a cross-sectional GIS study based on the Norwegian mother and child cohort study," BMC Public Health, vol. 19, no. 1, pp. 1-22, 2019.

[31] P. Carthy, S. Lyons, and A. Nolan, "Characterising urban green space density and footpath-accessibility in models of BMI," BMC Public Health, vol. 20, no. 1, pp. 1-12, 2020.

[32] Y. Xu, Y. Guo, A. K. Jumani, and S. F. A. Khatib, “Application of ecological ideas in indoor environmental art design based on hybrid conformal prediction algorithm framework," Environmental Impact Assessment Review, vol. 86, Article ID 106494, 2021.

[33] J. Yang, J. Zhang, and H. Wang, "Urban traffic control in software defined internet of things via a multi-agent deep reinforcement learning approach," IEEE Transactions on Intelligent Transportation Systems, pp. 1-13, 2020. 